\title{
LOS MILLENNIALS PERUANOS: CARACTERÍSTICAS Y PROYECCIONES DE VIDA
}

\author{
PERUVIAN MILLENIALS: FEATURES AND PROJECTIONS OF LIFE
}

José D. Begazo Villanueva* d180045@mixmail.com

Walter Fernandez Baca**** wfernandezbaca@pucp.pe

[RECEPCIÓN: AGOSTO 2015 / CONFORMIDAD: NOVIEMBRE 2015]

\section{RESUMEN}

El presente documento aborda el tema de la llamada Generación Millennials o Generación Y, que comprende los nacidos entre 1981 y el año 2,000. Esta generación ha revolucionado y cambiado los hábitos de la sociedad de consumo con respecto a otras generaciones, modificando las estrategias de mercado de las corporaciones especialmente en su potencial de marca llamado también Branding.

Actualmente, esta generación tiene entre 16 y 35 años, según el Centro Nacional de Planeamiento Estratégico- CEPLAN PERU (Diario Gestión 4 Marzo 2015) en los siguientes 10 o 15 años la PEA del Perú estará fundamentalmente cargada de personas que pertenecen al grupo de los llamados Millennials, ya que serán alrededor de 7 millones de personas; otro dato importante es que para el 2025 al 2030, más del 60\% de la fuerza laboral estará conformado por nativos digitales; es decir por millennials y post millennials.

Los Millennials peruanos son grandes seguidores de las tecnologías, y uno de sus símbolos, incluso mayor que el portátil o la televisión, es su Smartphone. Lo utilizan para navegar por internet, los de mejor posición económica realizan compras online y generan contenido en sus redes sociales, etc. Siempre están deseando compartir sus experiencias, aquello que no se comparte no tiene valor ni ha existido nunca.

El concepto de Millennials en el Perú está ligado a los millennials de Europa y Estados Unidos, la consultora en Recurso Humanos ER Ronald realizó una investigación donde establece el parecido de los millennials occidentales con las clases A, B y C altas peruanas. A raíz de esta investigación vamos a proponer estrategias de valor a ser utilizado por las organizaciones que tengan como visión un universo dirigido por los Millennials.

Palabras clave: Millennials, generaciones, proyecciones de vida, retroalimentación.

* Profesor Principal de la Facultad de Ciencias Administrativas (FCA) de la Universidad Nacional Mayor de San Marcos (UNMSM).

*** Profesor de la Pontificia Universidad Católica del Perú y de la Universidad Nacional Mayor de San Marcos (UNMSM). 


\begin{abstract}
This paper addresses the issue of the Millennials Generation or Generation Y, comprising those born between 1981 and 2000. This generation has revolutionized and changed the habits of the consumer society compared to other generations, changing market strategies of large corporations especially in its potential brand also called Branding. Currently, this generation is between 16 and 35 years, according CEPLAN Peru in the next 10 or 15 years the PEA of Peru will be charged mainly by people belonging to the group of so-called Millennials, as they will be around 7 million people; Another important fact is that by 2025 to 2030 , over $60 \%$ of the labor force will consist of digital natives; ie by millennials and post millennials.

Peruvian Millennials are big fans of technology, and one of its symbols, even greater than the laptop or TV, is their Smartphone. They use it to surf the internet, the better-off making purchases online and generate content on their social networks, etc. They are always willing to share their experiences, what has not shared has no value and never has existed.

The concept of Millennials in Peru is linked to millennials Europe and the United States, Human Resource consultant Ronald ER conducted an investigation which established the similarity of the Western millennials with classes A, B and C Peruvian high. Following this paper we propose strategies of value to be used by organizations having as view an universe led by Millennials.
\end{abstract}

Keywords: Millennials, generation, projected life and feedback.

\section{INTRODUCCIÓN}

En la coyuntura actual, estamos conviviendo con una generación única: Los Millennials, una generación nativa digital que abraza la economía colaborativa y duerme con el teléfono inteligente o la tableta cerca de la cama. El objetivo de este trabajo no es determinar las diversas características de todas las generaciones que han evolucionado en las últimas décadas, sino crear una proyección de esta generación en los próximos años, cuales son los retos a tener en cuenta para una mejor transferencia de poder en un futuro cercano; es lanzar propuestas para mejorar las prácticas de relaciones humanas mediante políticas a ser aplicadas en la generación Millennials.

Resulta inevitable que el comportamiento como consumidores de los millennials sea distinto al de sus padres. Los millennials quieren tener relaciones abiertas y honestas con sus gerentes y colegas, las empresas lo saben y tratan de leer sus pautas, "los millennials están acostumbrados a vivir fuera del anonimato y comparten en las redes sociales sus gustos y criterios" (Diario El País 2015/07/09 España).

Otra característica es su "infidelidad hacia las marcas". Enfrentados a una generación con criterios tan borrosos, ¿de qué forma pueden las organizaciones lucrativas y no lucrativas atraer su mirada? Para que un Millennials tenga productividad necesitan un ambiente laboral óptimo, con salas de reuniones agradables; espacios abiertos que inviten a la colaboración de ideas.

No hay fechas precisas de cuando esta generación comienza y termina. Los investigadores y comentaristas usan años de nacimiento que van desde la década de 1980 hasta principios del 2000.

\section{GENERACIONES DIVERSAS}

En nuestra sociedad actual y en las organizaciones de todo tipo observamos tres generaciones distintas trabajando juntas. ¿Qué tal se llevan estas diferentes generaciones en el ámbito de las relaciones humanas? Las organizaciones y oficinas de hoy reúnen tres generaciones de trabajadores. Lejos de significar un problema, por la brecha de años, logran que los Millennials, los Baby Boomers y la Generación X se complementen y aprendan entre sí. (Revista FORBES estudio de la Benefits for Tomorrow Study Julio 2013)

Llamamos Generación Y o Millennials a los empleados jóvenes que agrupamos entre los 18 y los 35 años. La generación $\mathrm{X}$ abarca a los que tienen entre 36 y 49, y los Baby Boomers comprenden a los que tienen entre 50 y 60 .

Trabajar cooperativamente con personas de otra generación es un factor clave para aprender. Así los Baby Boomers pueden transmitir a la gene- 
ración siguiente su experiencia, y los Millennials pueden aportar una forma de trabajo innovadora que estimule un mejor desempeño.

Se crea un cuadro multigeneracional aun cuando no se cuente con un programa específico o un código de trabajo para las diferentes generaciones, resulta beneficioso reunir a miembros de distintas edades para poner en común diversos intereses y perspectivas que mejoren el trabajo diario.

Las tres generación de acuerdo a su edad son: Baby Boomers, Generación X y Millennials, Cada generación tiene diferentes características y maneras de interpretar la vida y felicidad (Molinari 2011) nosotros coincidimos e indicamos que son completamente distintas una de la otra.

\section{Baby Boomers}

Para muchos este nombre resulta extraño e incluso despectivo, ya que hace referencia a los adultos mayores. Es el segmento que considera el rango de edad más amplio y su nombre es resultado del boom de nacimientos que se dio durante el segundo y tercer cuarto del siglo XX y hace referencia a personas nacidas entre 1933 y 1960, aunque la oficina de Censos de USA considera a los Baby Boomers como los nacidos entre 1946 y 1964. A esta generación se le llama de varias formas dependiendo la connotación, dos de las más conocidas son Alpha Boomers y Golden Boomers haciendo referencia a que en los 90's y a principios de este siglo, muchos de los líderes mundiales pertenecían a esta generación.

Los Baby Boomers en Perú y América Latina son ligeramente distintos a los de USA, ya que a esta generación les tocó vivir las revoluciones, crisis y devaluaciones, por lo cual las palabras "Inflación", "Deuda Externa” y "Crisis" les son muy familiares, los impulsan a cuidar el dinero e incluso a buscar contar con inmuebles propios en lugar de rentar, para sentir certidumbre económica hacía el futuro.

Su periodo económicamente más productivo se dio entre las décadas de los $70^{\prime}$ 's y $90^{\prime}$ s, y aunque todavía actualmente hay un gran número de ellos trabajando, la mayoría ya se encuentran jubilados o en proceso de hacerlo. Para esta generación el matrimonio heterosexual es el camino para conformar una familia, y de las generaciones actuales, es la más apegada a la religión. Acostumbran ver, escuchar o leer noticias, por lo que es común encontrarlos leyendo un periódico impreso o viendo un noticiero en la TV.

A nivel tecnológico a los Baby Boomers les tocó vivir el nacimiento de la televisión, vieron en vivo la llegada del hombre a la luna, pasaron del cine en blanco y negro al de color, del teléfono de disco al de tonos, se maravillaron con la llegada del fax, la lavadora eléctrica, los relojes de pulsera digitales, y por si no fuera suficiente para ellos, también vieron nacer a la telefonía celular, la computadora personal y el Internet. En cuanto al entretenimiento, como generación en la TV les gustan los programas de concurso, los noticieros y los documentales. Son la generación que más lee, principalmente novelas y libros de superación personal. Si bien disfrutan salir de su casa, por lo general son más hogareños y prefieren comer en casa a pagar por hacerlo fuera.

\section{Generación X}

Esta generación que hoy es la que mueve al mundo a nivel laboral y económico, obtiene su nombre gracias a la dificultad de la generación anterior para entenderla, llamándola generación $\mathrm{X}$, porque a su entender no tenía rumbo ni sentido. La Gen X como también se le dice, considera a los nacidos entre 1960 y 1984, los cuales actualmente tienen entre 30 y 54 años y son hijos de los Baby Boomers y padres de los Millenials. A esta generación se le llama de varias formas, las más populares son Generación MTV, La Generación X a diferencia de los Baby Boomers si se considera el ser emprendedor, gracias a que su nivel educativo es mayor que el de la generación previa y a la rebeldía que muestra hacia modelos anteriores de negocio. Los Gen X son los grandes impulsores de la tecnología, ya que nacieron justo en el boom en el que se dejó de hablar de aparatos eléctricos para hablar de dispositivos electrónicos, pero aunque gustan por la tecnología, al segmento más longevo de esta generación, el internet, las redes sociales y el comercio electrónico todavía les resulta ajeno.

A esta generación le toco vivir el nacimiento de Internet y la burbuja del .com en la década de los 90 's, por eso actualmente parte de esta generación se resiste a utilizar estas tecnologías. La familia es todavía un valor como generación, y se muestran mucho más abiertos a la diversidad sexual, de raza y política. A nivel religioso, siguen profesando una religión, pero no son tan devotos y constantes como las generaciones previas. 
A nivel educativo, están preocupados por su preparación, y buscan en la medida de sus posibilidades estudiar algo más que solo la universidad, por esta razón los MBA se han vuelto tan populares. A nivel social, esta generación busca mostrar su éxito por medio de propiedades, gadgets y accesorios, así como en restaurantes, bares y viajes.

\section{Los millennials}

También llamados Generación Y es la generación más joven, conformada por personas nacidas a partir de 1984, así que al día de hoy tienen menos de 35 años de edad. Este grupo es el más complicado de describir en general, ya que considera a niños, adolescentes y adultos de menos de treinta años, aunque sorprendentemente existen varias similitudes. Esta generación se declara completamente liberal en todos los sentidos, desde el punto de vista familiar no consideran el matrimonio como una opción, encuentran el tener hijos un tema más para el futuro y las relaciones entre parejas del mismo sexo son bien vistas y aceptadas. A nivel educativo, esta generación prefiere aprender de manera autodidacta, de ahí que los tutoriales en internet sean su principal fuente de conocimiento.

Esta generación nació con Internet, por lo cual es su principal herramienta para distintos ámbitos de su vida; desde la comunicación por medio de redes sociales, la educación por medio de podcast, vida podcast y video blogs, hasta el entretenimiento por medio de video juegos y canales de video en línea.

Si bien las computadoras son la herramienta favorita de la Generación X, para los millennials son los smartphones y tablet, y pasan gran parte de su tiempo utilizando estos dispositivos durante el día. Son grandes consumidores de video en Internet, ya que les permite contar con el contenido cuando lo desean, situación que los ha alejado de la TV y la radio.

Esta generación prefiere trabajar desde casa, no se siente cómoda con los horarios de oficina, por eso busca opciones de Home Office o en su caso son emprendedores que buscan abrir sus propias empresas. Por lo general duran poco tiempo en un empleo gracias a las grandes expectativas que generan hacía el trabajo. A nivel cultural, son una generación que no le interesan los medios de comunicación, por lo que las noticias llegan a su vida de manera viral en redes sociales.
Es la generación que menos lee y aunque tienen un gran sentido social, no buscan profundizar en la información y se quedan con lo que les llega. A nivel social, la mayoría no profesa una religión y por lo general prefieren a nivel político opciones de izquierda. Son idealistas y sueñan con hacer grandes cosas en su vida, así como dejar un impacto en la sociedad. Esta generación tiene en la cabeza temas ecológicos, de cuidado del medio ambiente y aunque en su aspecto personal podrían ser descuidados, si buscan comer de manera saludable y balanceada, de ahí que a esta generación en países occidentales algunos le llaman la generación Vegana.

\section{LAGENERACION MILLENNIAL PERUANA ¿QUIENES SON? ¿QUE PIENSAN?}

Se les dice millennials a aquellas personas nacidas entre los años 1981 y 2000, y que en la actualidad tienen por lo tanto entre 15 y 34 años. Internet ha "atravesado" su vida desde la niñez o la adolescencia, y por lo tanto su cosmovisión de lo que sucede está fuertemente influenciada por la tecnología y la era digital. Los millennials mayores (es decir, los nacidos en los ' 80 ) son hijos de los Baby Boomers (generación post-guerras mundiales), mientras que los menores (los nacidos a partir de los ‘90) son descendientes de la llamada generación $\mathrm{X}$.

Según encuestas realizadas para la publicación en SEMANA ECONÓMICA en conjunto con IPSOS APOYO, (la primera encuesta sobre la generación millennials a nivel nacional) el dinero y la estabilidad laboral sí interesa a la mayoría de millennials peruanos. La informalidad y lenta penetración de la tecnología coadyuvarían a que las generaciones peruanas demoren en cambiar sus características. Se dice que esta generación también llamada Y, quieren un trabajo con trascendencia, les gusta le flexibilidad laboral y el cambio de empresas es muy normal en ellos. Resulta que de toda la generación Y peruana equivale al 35\% de la población nacional, solo los más jóvenesaquellos entre 18 y 25 años y de mayor poder adquisitivo ostentan las características comúnmente asociadas a millennials.

La encuesta rompe un primer mito al concluir que a la generación Y sí le importa el dinero. Los resultados señalan que por dinero un millennial se queda en un centro laboral y que el nivel de sueldo es uno de los tres principales factores que le interesa al momento de buscar un trabajo. 
Para un millennial peruano el desarrollo profesional pleno se relaciona con hacer lo que le apasiona y más que ver con convertirse en un experto en su campo.

La encuesta también concluye que la generación Y peruana está dispuesta a trabajar horas extra a fin de ascender más rápido.

En lo que respecta a los millennials como consumidores, éstos no son tan distintos como sus predecesores, pues también se fijan en el precio y la calidad antes de elegir una marca. Sin embargo, existen factores que son importantes para la generación Y que no lo eran para la generación $\mathrm{X}$ y los baby boomers.

La percepción que terceros tienen de una marca es mucho más importante para los millennials que para generaciones pasadas. La encuesta muestra que las recomendaciones de especialistas u otros son altamente consideradas. No solo en lo que respecta a productos tecnológicos, donde las demás generaciones también buscan recomendaciones, sino también en productos de cuidado personal.

A ello se suma que la marca sea única y diferente. Este aspecto resulta prioritario para los millennials al momento de serle leal a una marca o no. Ello toda vez que la generación Y valora mucho la individualidad.

La razón detrás del desfase de la generación Y peruana responde a ciertas singularidades del Perú. Por ejemplo, la lenta penetración de la era digital, a junio de 2014 solo $21.4 \%$ de peruanos tiene acceso a un smartphone, según el Osiptel.

El ejecutivo explica que esta mayor juventud en la población se da en un contexto donde las empresas están creciendo y requieren llenar jefaturas y gerencias, pero no tienen las personas a las que estaban acostumbradas a contratar en dichos puestos. Así, muchos jóvenes han ascendido de manera rápida para ocupar estos puestos.

A ello se suma el tema de la tecnología. Un joven que puede estar en desventaja para ocupar un puesto por el número de años que tiene en la empresa, tiene una ventaja en sus conocimientos y manejo de tecnología.

"La convivencia intergeneracional en América Latina no es solo vertical, entonces, como en Estados Unidos, sino horizontal también. Ello genera la necesidad ya no solo de comprender a los empleados, sino de comprender a los pares para poder trabajar en equipo" (Rodolfo Arellano 2014).

En el presente año, los Baby Boomers más jóvenes tienen 52 años, estando cercanos a salir de la población económica activa (PEA). Entramos al declive de un largo reinado de esta generación. Y, más bien, entrando a un próximo mundo gobernado por los millennials en todos sus ámbitos: político, económico, cultural y social. En este sentido, es importante comprender y saber capacitar a esta nueva generación para su próximo trono empresarial y, por ende, social (Thalía Penagos y Ernesto Rubio - ER Ronald 2014).

\section{CARACTERÍSTICAS DE LOS MILLENNIALS}

$\checkmark$ No hay vida sin Internet: Para los Baby Boomers fue la radio. Para la generación $X$, la televisión. Para los millennials, en su momento fue la computadora de escritorio, luego la notebook y actualmente los Smartphone, pero las personas de las que estamos hablando no conciben la relación con el mundo sin estos dispositivos y la mayor parte de lo que hagan (desde mirar películas hasta trabajar o conocer gente o comprar productos) será siempre usando la última tecnología existente.

$\checkmark$ Visión carpe diem: Ponderan el presente por sobre todas las cosas, quieren vivir el momento actual intensamente y buscan la felicidad y la pasión en todo lo que hacen. Esto genera un cambio importante, ya que mientras que para las generaciones anteriores el sinónimo del éxito estaba siempre más relacionado con las posiciones jerárquicas en lo laboral, los millennials ponderan el trabajar de lo que les gusta por sobre el ejercicio del poder o incluso lo material.

$\checkmark$ Movilidad laboral: Cuando un millennial siente que su trabajo ya no presenta un reto, lo dejará sin remordimientos y buscará otro en donde se sienta más a gusto. También, dado que suelen ser multifacéticos (hacen varias cosas al mismo tiempo), es probable que tengan varios trabajos free-lance simultáneos. Actualmente, en USA se estima que un joven cambia 15 veces de trabajo antes de cumplir los 38 años. Eso demuestra el poco apego que existe para esta generación en lo referente a lo laboral.

$\checkmark$ Participación y crítica: Los millennials son participativos y les gusta compartir sus conocimientos. No creen demasiado en los derechos de autor y están a favor de democratizar la 
información y el contenido de manera gratuita. Al mismo tiempo, son desconfiados de lo que las empresas les dicen (sobre todo las grandes multinacionales) y chequean e investigan en la web antes de tomar cualquier decisión de consumo.

$\checkmark$ Poca posesión, mucho disfrute: No les interesa el «sueño de la casa propia». Tampoco se mueren por tener su propio auto. Algunos hasta prefieren alquilar la ropa que usan en vez de comprarla (porque no es nada bueno aparecer en las fotos de Instagram o Facebook siempre con la misma ropa). Los millennials tampoco son de acumular bienes y, como veremos más adelante, el único gasto que vale la pena tiene que ver con la compra del último modelo de Smartphone. Privilegian el disfrute, el viajar y la inversión en "experiencias" por sobre la posesión física de las cosas, cualesquiera que sean.

\section{PROYECCIONES DE VIDA Y COMO VALORAR A LOS MILLENNIALS PERUANOS}

Las organizaciones y corporaciones peruanas deben de preocuparse en el perfil descrito anteriormente: Jóvenes que tienen ganas de crecer en el trabajo, "Si una empresa no le da a sus empleados jóvenes la oportunidad de crecer, aprender y desarrollarse rápido en la organización, simplemente se van a otra organización”, indica Rolando Arellano, un doctrinario del marketing en América Latina.

Los millennials no quieren que una empresa les diga que no se merecen un puesto o una remuneración solo porque son jóvenes. Quieren ser evaluados en base a habilidades y resultados. "Hoy en día, el criterio de antiguiedad se podría dejar de lado".

Tratar con la generación del nuevo milenio no debe ser como si no se pudiera ni tocarlos con el pétalo de una rosa. Todo el mundo sabe que a los llamados Millenials hay que darles continua retroalimentación, que requieren fuerte liderazgo y constante aliento de parte de los gerentes.

Estos voraces usuarios de las nuevas tecnologías también esperan que sus jefes les provean horarios flexibles y rienda suelta para trabajar desde casa. Más aún, un reciente estudio del Centro de Investigación PEW en marzo 2015, en Estados Unidos, reveló que $83 \%$ de los miembros de la generación del milenio encuestados duer- men teniendo cerca su smartphone, un porcentaje mucho más alto en relación con sus padres o abuelos. Pero satisfacer las demandas en el lugar de trabajo de los profesionales millennials de entre 18 y 29 años no tiene por qué ser oneroso. Las propuestas para dar valor son:

$\checkmark$ Conceder acceso instantáneo. La mayoría de la nueva generación necesita sentir que está contribuyendo a la organización. El problema estriba en que muchas compañías aplican un lento proceso de aclimatación que puede durar semanas de orientación y entrenamiento.

Esto es un error, "La generación del milenio quiere participar en la organización desde un comienzo, Por desgracia, a menudo se les dice que necesitan tiempo para ser parte de la cultura de la empresa. Pero la realidad es que la mayoría de los Millenials siente que tiene por delante una misión que cumplir: quieren poner manos a la obra desde el primer momento".

$\checkmark$ Volcarse en elogios. En los viejos tiempos uno sabía que estaba realizando un buen trabajo si tenía la suerte de tenerlo. Pero la nueva generación requiere de constante retroalimentación y el visto bueno de los jefes, cuando alguien está haciendo algo mal, la nueva generación requiere apoyo instantáneo. No hay que estar encima de ellos todo el tiempo, pero si el jefe recibe un mensaje de texto, tiene que contestarlo. Uno debe estar siempre dispuesto a responder y hay que estarles repitiendo una y otra vez que está bien lo que están haciendo.

$\checkmark$ Dar libertad. Además de que los millenials son ligeramente de 'alto mantenimiento', las expectativas de sueldo de dicha generación suelen ser realistas y rara vez se salen de presupuesto con respecto a las tendencias del mercado laboral. "Las aspiraciones de la generación del milenio son diferentes. Se sienten cómodos con ese equilibrio de su vida laboral y trabajar con otra gente".

$\checkmark$ Retribuir a la comunidad. Los cursos de entrenamiento totalmente pagados y los aguinaldos en Navidad pueden inclinar a los jóvenes millenials a quedarse a bordo. No obstante, nada se compara con dar a estos veinteañeros la oportunidad de retribuir a la comunidad y comprometerse con el trabajo filantrópico. 


\section{CONCLUSIONES}

1. No existen fechas precisas cuando esta generación comienza y termina. Los expertos usan años de nacimiento que van desde la década de 1980 hasta principios del año 2000.

2. El organismo del gobierno peruano: CEPLAN ha proyectado que en los siguientes 10 o 15 años la PEA del Perú estará cargada de personas millennials, ya que serán alrededor de 7 millones de personas; también indican que para el 2025 al 2030, más del 60\% de la fuerza laboral estará conformado por nativos digitales; es decir, por millennials y post millennials.

3. Las tres generaciones estudiadas son: Baby Boomers, Generación X y Millennials, cada generación tiene diferentes características e idiosincrasias, son completamente distintas una de la otra.

4. Se les dice millennials a aquellas personas las cuales el Internet ha "atravesado" su vida desde la niñez o la adolescencia, y por lo tanto su cosmovisión de lo que sucede está fuertemente influenciada por la tecnología y la era digital. Los millennials mayores (es decir, los nacidos en los '80) son hijos de los Baby Boomers (generación post-guerras mundiales), mientras que los menores (los nacidos a partir de los '90) son descendientes de la llamada Generación X.
5. "La convivencia intergeneracional en América Latina no es solo vertical, sino horizontal también. Ello genera la necesidad ya no solo de comprender a los empleados, sino de comprender a los pares para poder trabajar en equipo".

6. A los llamados millenials hay que darles continua retroalimentación, requieren fuerte liderazgo y constante aliento de parte de los líderes. Estos jóvenes usuarios de las nuevas tecnologías también esperan que sus jefes les provean horarios flexibles y libertad para trabajar desde casa.

\section{REFERENCIAS BIBLIOGRÁFICAS}

Arellano, Rolando (2013): Marketing para Vivir Mejor. Editorial Planeta, Lima.

Centro Nacional de Planeamiento EstratégicoCEPLAN PERU. Los Millennials - Características (Artículo del Diario Gestión 4 Marzo 2015 - Lima-Perú).

Molinari P. (1996). "Turbulencia Generacional”. Temas Grupo Editorial. Buenos Aires.

Ortiz de Zevallos, Fernando (2013). "Los hábitos de la próxima generación”. Revista Semana Económica. N. ${ }^{\circ} 18$, Lima - Perú.

Thalía Penagos \& Ernesto Rubio (2015). "Millenials y Millennials peruanos". Editorial Ronald, Lima. 